\title{
COLONIZAÇÃO NASAL E EM OROFARINGE POR STAPHYLOCOCCUS AUREUS EM CRIANÇAS E ADOLESCENTES DE UM BAIRRO DO MUNICÍPIO DE VITÓRIA DA CONQUISTA, BAHIA, BRASIL
}

\author{
NASAL AND OROPHARYNGEAL COLONIZATION BY STAPHYLOCOCCUS \\ AUREUS IN CHILDREN AND ADOLESCENTS FROM A NEIGHBORHOOD IN \\ VITÓRIA DA CONQUISTA, BAHIA, BRAZIL
}

\author{
Milena Soares dos Santos, Jefferson Nilton Silva dos Santos, Sabrina Santos Alves, André \\ Brito Novais, Verônica Cheles Vieira
}

Universidade Federal da Bahia

\begin{abstract}
Staphylococcus aureus is a Gram-positive bacterium that inhabits the nasal cavity of about $30 \%$ of the healthy population. Antimicrobial resistance has emerged in the community and has become a global concern. The aim of this study is to determine the prevalence of nasal and oropharyngeal colonization caused by this pathogen in healthy children and adolescents from a community in the city of Vitória da Conquista, Bahia and to evaluate the frequency of antimicrobial resistant isolates. This is a cross-sectional study, conducted through epidemiological and laboratory research based on interviews and obtaining swabs of nasal and oropharyngeal secretion. Microbiological identification was performed by conventional methods and the profile of antimicrobial susceptibility by disc-diffusion. The Epilnfo Windows version 3.5.4 program was used for statistical analysis of the data. A prevalence of $73.1 \%$ was identified for colonization by S.aureus in a nasal and/or oropharynx sample and of $31.2 \%$ in both anatomical sites. Among the isolates from the nasal sample, $26.7 \%$ were resistant to at least one antimicrobial and $6.7 \%$ were methicillin-resistant. For oropharyngeal samples, $35.3 \%$ of non-susceptibility to erythromycin was identified. A high rate of nasal and oropharyngeal colonization by S. aureus was observed among the analyzed participants. Thus, we highlight the importance of continuous monitoring of this pathogen and its resistant variants in the community.
\end{abstract}

Keywords: Staphylococcus aureus; carrier state; drugs resistance, microbial

\section{Resumo}

Staphylococcus aureus é uma bactéria Gram-positiva que habita a cavidade nasal de cerca de 30\% da população saudável. A resistência aos antimicrobianos tem emergido na comunidade e se tornado uma preocupação global. O objetivo deste estudo é determinar prevalência de colonização nasal e de orofaringe causada por este patógeno em crianças e adolescentes saudáveis de uma comunidade na cidade de Vitória da Conquista, Bahia e avaliar a prevalência de isolados resistentes aos antimicrobianos. Trata-se de estudo transversal, conduzido através de investigação epidemiológica e laboratorial a partir de entrevistas e obtenção de swabs de secreção nasal e de orofaringe. A identificação microbiológica foi realizada por métodos convencionais e o perfil de susceptibilidade antimicrobiana por disco-difusão. $O$ programa Epilnfo Windows versão 3.5.4 foi utilizado para análise estatística dos dados. Identificou-se prevalência de $73,1 \%$ para colonização por S.aureus em amostra nasal e/ou orofaringe e de $31,2 \%$ em ambos os sítios anatômicos. Entre os isolados de amostra nasal, 26,7\% apresentaram resistência a pelo menos um antimicrobiano e 6,7\% foram resistentes à meticilina. Para amostras de orofaringe, identificou-se $35,3 \%$ de não susceptibilidade à eritromicina. Foi observada alta taxa de colonização nasal e orofaríngea por S. aureus entre os participantes analisados. Dessa forma, destacamos a importância do contínuo monitoramento deste patógeno e de suas variantes resistentes na comunidade.

Palavras-chave: Staphylococcus aureus; portador sadio; resistência microbiana a antibióticos. 
Staphylococcus aureus é uma bactéria Gram-positiva capaz de habitar vários nichos do corpo humano, como pele, mãos, axilas, períneo e cavidades oral e nasal, sendo esta última seu principal habitat ${ }^{1,2,3}$. A colonização representa uma linha tênue entre um processo comensalista e um prejudicial ao hospedeiro, sendo este equilíbrio influenciado por fatores intrínsecos do micro-organismo e do próprio individuo ${ }^{4}$.

Essa bactéria pode habitar a cavidade nasal de cerca de $30 \%$ da população saudável, mas também é responsável por diversas doenças humanas, que variam das mais leves, como algumas piodermites (impetigo, furúnculo, celulite e espinhas) às mais graves (endocardite, sepse, pneumonia nosocomial e síndrome do choque tóxico $)^{3,4,5,6}$. Os indivíduos colonizados por S.aureus geralmente mantém-se assintomáticos, porém são capazes de disseminar a bactéria a outras pessoas ${ }^{7,8}$.

Quanto à orofaringe, ainda não está completamente elucidado se $S$. aureus é colonizador nativo desse microbioma ou se o habita transitoriamente. Sabe-se da sua participação em afecções como: queilite angular, osteomielite mandibular e infecções endodônticas ${ }^{9}$.

Os mecanismos de patogenicidade estão intimamente relacionados com sua capacidade de virulência, que vão desde a fixação, penetração e multiplicação até a interação com o sistema imunológico do hospedeiro ${ }^{10}$. Proteínas MSCRAMMs (do inglês, Microbial Surface Components Recognizing Adhesive Matrix Molecules) são responsáveis pela interação da superfície microbiana com a matriz do hospedeiro, desempenhando um importante papel na colonização por estes microorganismos $^{11}$.

S.aureus também produz a hialuronidase e apresenta outros componentes estruturais, como os glicanopeptídeos, o ácido teicóico e os lipídios $A$ e cápsula antifagocitária que se relacionam intimamente com o sistema imunológico do hospedeiro. Este, ao ser estimulado, produz citocinas e interleucinas próinflamatórias, como a IL-1, que são responsáveis pela imunidade inata, com o protagonismo das células polimorfonucleares, que geralmente são responsáveis pela sintomatologia apresentada pelos infectados. Concomitantemente, esses componentes estruturais também são responsáveis por evadir esta mesma resposta imunológica, possibilitando a sobrevivência do micro-organismo, a exemplo da Proteína A que ao se ligar à porção $F c$ das imunoglobulinas $G$ $(I g G)$, compromete a resposta humoral e, consequentemente, os estímulos quimiotáticos e fagocitários $^{10,11}$.

Outra importante característica patogênica é a produção de toxinas, como a esfoliatina, responsável pela "síndrome da pele escaldada" e a TSST-1, uma exotoxina classificada como superantígeno capaz de ativar os linfócitos $\mathrm{T}$, estimulando-os a produzir uma quantidade excessiva de citocinas (dentre elas IL-1, TNF- $\alpha$ e IL-2) causando uma reação autoimune reduzindo as populações dessas células por morte de seus clones $^{10,12}$

Destaca-se também como fator de virulência, a capacidade dessas bactérias de formarem biofilmes, que possuem uma íntima relação com o aumento da resistência a múltiplos antibióticos. $O$ gene icaABCD é responsável pela adesão intercelular de polissacarídeos (PIA) através da expressão do poli- $\beta(1-6)-N$ acetilglucosamina, um dos componentes mais importantes para a formação de biofilmes. Estudos apontam uma maior prevalência do icaD e icaA em bactérias constituintes de biofilmes fortes $^{11,13}$

Outra preocupação global se refere aos mecanismos de resistência aos agentes antimicrobianos utilizados nos tratamentos de suas afecções. Os isolados de $S$. aureus resistente à meticilina (MRSA) são codificados através do gene mecA, localizado em uma estrutura genética móvel denominado cassete estafilocócico cromossômico (SCCmec). Este expressa a PBPa2 (proteína de ligação a penicilina) que possui uma baixa atração a penicilina. Acredita-se que, de forma evolutiva, estas bactérias espessaram a sua parede celular, impedindo a chegada do antibiótico até a membrana citoplasmática, seu local de atuação ${ }^{3,10,13}$.

Cepas MRSA foram identificadas em $17,4 \%$ de amostras das cavidades nasal e/ou orofaríngea de crianças em idade escolar da Nova Zelândia ${ }^{14}$. Em um estudo brasileiro, cerca de um terço de todas as cepas de $S$. aureus obtidas de crianças com infecções comunitárias graves eram MRSA (CA-MRSA). Um aumento gradual na presença dessas bactérias resistentes em relação às cepas hospitalares (HA-MRSA) foi relacionado a um maior perfil de casos de letalidade ${ }^{15}$. 
Considerando a relevância de S.aureus no cenário clínico, visto que é um dos principais organismos comensais dos seres humanos com alto potencial e habilidade de virulência, este estudo tem como objetivo determinar a prevalência de colonização nasal e de orofaringe por S.aureus em crianças e adolescentes saudáveis de uma comunidade do município de Vitória da Conquista, Bahia, Brasil e a prevalência de isolados resistentes aos antimicrobianos nessa população.

\section{Metodologia}

\section{Aspectos éticos}

Esta pesquisa faz parte do projeto intitulado: "Projeto de extensão: Endemias e Meio Ambiente em Vitória da Conquista, Bahia" conduzido conforme a Resolução do Conselho Nacional de Saúde 466 de 12 de dezembro de 2012 do Ministério da Saúde, Brasil, aprovado como emenda pelo comitê de ética em pesquisa em seres humanos do Instituto Multidisciplinar em Saúde, Campus Anísio Teixeira, Universidade Federal da Bahia, aprovado sob parecer $n^{\circ}$ 2.384.442. O projeto está cadastrado na plataforma SisGen - Sistema Nacional De Gestão Do Patrimônio Genético e do Conhecimento Tradicional Associado em atendimento ao previsto na Lei no $13.123 / 2015$ e seus regulamentos sob registro $A E A 2 E B E$.

\section{Desenho, local e período do estudo}

Trata-se de um estudo de corte transversal, com base em dados epidemiológicos, clínicos e laboratoriais, abordagem descritiva e quantitativa. Foi conduzido em uma associação sem fins lucrativos denominada Projeto Resgatados, gerenciada pela Igreja Evangélica Candeias-Congregação, na comunidade do Bairro Primavera, localizado na zona urbana de Vitória da Conquista, município localizado no sudoeste baiano, terceira maior cidade do estado, com uma população de 341.597 habitantes, segundo estimativa para $2019^{16}$.

O período do estudo correspondeu a maio de 2017 a julho de 2019. O Laboratório de Análises Clínicas do Instituto Multidisciplinar em Saúde, Campus Anísio Teixeira, Universidade Federal da Bahia (IMS-CAT-UFBA) foi o local selecionado para processamento e análise laboratorial microbiológica, procedimentos de identificação, armazenamento de amostras, criação e manutenção de banco de dados para as análises.
População do estudo

A população do estudo foi composta por amostragem de conveniência onde foram convidados crianças e adolescentes incluídos no projeto Resgatados. Indivíduos que realizaram uso prévio de antimicrobianos e os que não estiveram em jejum por pelo menos $2 \mathrm{~h}$ antes da coleta de amostra de orofaringe foram excluídos do estudo.

Coleta de dados, amostra biológica e análise microbiológica

Foram realizadas entrevistas com os responsáveis legais das crianças e adolescentes para as informações epidemiológicas e clínicas. As amostras nasais e de orofaringe foram obtidas através de swabs com ponta de algodão estéreis, flexíveis, que foram acondicionados em caldo STGG (SkimMilk, Triptyc Soy Broth, Glicose e Glicerol) para transporte e criopreservação. Os swabs foram homogeneizados em vórtex. Na sequência, uma alíquota de aproximadamente 20 $\mu \mathrm{L}$ foi semeada em placa contendo ágar-salmanitol (Himedia ${ }^{\circledR}$ ) e incubada em estufa bacteriológica a $35^{\circ} \mathrm{C}+/-2^{\circ} \mathrm{C}$ por 18 a $24 \mathrm{~h}$. As colônias com características típicas de $S$. aureus foram selecionadas e semeadas novamente em ágar nutritivo (Accumedia ${ }^{\circledR}$ ) para realização dos testes de identificação através de métodos convencionais, como coloração de Gram, catalase, coagulase em lâmina e posterior criopreservação em caldo BHI (Brain Heart Infusion Broth, Himedia ${ }^{\circledR}$ ) acrescido de glicerol até finalização dos testes.

O perfil de susceptibilidade dos isolados foi realizado através do método de disco difusão em ágar Mueller Hinton (Beckton-Dickinson, Difco $\left.^{\circledR}\right)$. Os antimicrobianos cefoxitina $(30 \mu \mathrm{g})$, clindamicina $(2 \mu \mathrm{g})$, eritromicina $(15 \mu \mathrm{g})$, gentamicina $(10 \mu \mathrm{g})$, tetraciclina $(30 \mu \mathrm{g})$, sulfametoxazol-trimetropim $(1,25 / 23,75 \mu \mathrm{g})$ e cloranfenicol $\left(30 \mu \mathrm{g}\right.$ ) (Sensifar $\left.{ }^{\circledR}\right)$ foram utilizados e interpretados segundo recomendações do Clinical Laboratory Standards Institute, 2017. A detecção do fenótipo de resistência aos macrolídeos, lincosaminas e estreptogramina B (MLSB) foi realizada através do teste de aproximação dos discos eritromicina e clindamicina. A cepa de referência Staphylococcus aureus ATCC 25923 foi utilizada como controle.

Seleção e interpretação das variáveis 
Foram avaliadas informações sociodemográficas e clínicas dos participantes, incluindo as seguintes variáveis: idade, sexo, convívio com crianças, idosos e fumantes, comorbidades, dormir em aglomerados, sinais e sintomas clínicos nos últimos 30 dias, antibioticoterapia prévia e status vacinal. Para análise de cor da pele, foram considerados como Branca as crianças e adolescentes que foram autodeclarados pelos responsáveis legais como cor da pele branca e Não Branca para os indivíduos que intitularam a cor da pele da criança ou adolescente como preto, mulato ou pardo. Para análise da escolaridade dos pais, foram alocados em um grupo os indivíduos não alfabetizados e com Ensino Fundamental Incompleto (EFI) e os responsáveis com Ensino Fundamental completo (EFC), Ensino Médio Incompleto (EMI) ou Ensino Médio Completo (EMC) em outro grupo. Dormir em aglomerados foi considerado para os participantes que dormiam junto com duas ou mais pessoas na mesma cama. Foram considerados como sadios os participantes cujos responsáveis legais não relataram nenhum sinal ou sintoma clínico nos últimos 30 dias em relação ao momento da entrevista e como doenças prévias os que apresentaram e como portadores de comorbidades, aqueles cujos pais indicaram que apresentaram alguma doença ou condição prévia de agravo à saúde, crônicos ou desde o nascimento. Para cálculo do Índice de Massa Corporal (IMC) foram considerados altura, peso, sexo e idade do participante através da fórmula: $\mathrm{IMC}=$ Peso $\mathrm{kg} /(\text { altura } \mathrm{m})^{2}{ }^{17}$. A variável de desfecho foi considerada como a presença de colonização sendo dicotomizada em sim ou não.

\section{Análise de dados}

O programa Epilnfo Windows versão 3.5.4 (Center for Diseases Control and Prevention, Atlanta, Georgia, USA, 2017) foi utilizado para a criação e análise do banco de dados. Os dados foram interpretados através de análise descritiva, cálculos de média e frequência simples. As variáveis categóricas foram comparadas utilizando o teste exato de Fisher bi-caudal ou o qui-quadrado e valor de $p$ corrigido por MantelHaenszel. Neste estudo foram realizadas análises univariada e bivariada, sendo calculada Odds Ratio (OR) como medida de associação. Foram consideradas estatisticamente significantes as medidas com valor de $p<0,05$ e intervalo de confiança (IC) de $95 \%$.

\section{Resultados}

Um total de 67 crianças e adolescentes com idade entre 4 e 15 anos (Mediana 9 anos; $\left.1 Q_{25}=7-1 Q_{75}=12\right)$, sendo $n=42$ do sexo masculino $(62,7 \%)$ e $n=25(37,3 \%)$ do sexo feminino foram envolvidas no estudo, dos quais foram obtidas amostras das narinas de todos participantes e de orofaringe para 96\% (64/67) dos mesmos. Foi identificada prevalência de 73,1\% (49/67) para colonização por S.aureus em amostra nasal e/ou orofaringe, 67,2\% (45/67) para mucosa nasal, $37,5 \%$ (24/64) para orofaringe e 31,2\% (20/64) em ambos os sítios anatômicos. As características gerais das crianças e adolescentes colonizados e a análise estatística estão representadas na tabela 1.

Tabela 1 - Características epidemiológicas e clínicas de crianças e adolescentes sadios estratificadas pela colonização por Staphylococcus aureus em mucosa nasal e/ou orofaringe, Vitória da Conauista-Bahia

\begin{tabular}{lcccc}
\hline & \multicolumn{2}{c}{ Colonização por S.aureus } & Medidas estatísticas \\
\cline { 2 - 5 } Variáveis & Sim & Não & \\
\hline Sexo & $\mathrm{n}(\%)$ & $\mathrm{n}(\%)$ & OR (IC 95\%) & Valor de $\boldsymbol{p}$ \\
Feminino & $19(38,8)$ & $6(33,3)$ & $1,27(0,41-3,94)$ & 0,68 \\
Masculino & $30(61,2)$ & $12(66,7)$ & & \\
Idade (em anos) & & & & \\
04 a 11 & $43(87,8)$ & $15(83,3)$ & $1,43(0,32-6,46)$ & \\
$\geq 12$ & $6(12,2)$ & $3(16,7)$ & & \\
Cor & & & & \\
Branco & $14(28,6)$ & $3(16,7)$ & $2(0,50-7,99)$ \\
Não branco & $35(71,4)$ & $15(83,3)$ & &
\end{tabular}


Continuação...

\section{Convívio com outras crianças}

Sim

Não

\begin{tabular}{|c|c|}
\hline $22(44,9)$ & $7(38,9)$ \\
\hline $27(55,1)$ & $11(61,1)$ \\
\hline
\end{tabular}

\section{Convívio com idosos}

Sim

$\begin{array}{cc}2(4,1) & 0(0) \\ 47(95,9) & 0(0)\end{array}$

Não

Convívio com fumantes

Sim

$\begin{array}{lccc}15(30,6) & 4(22,2) & 1,54(0,43-5,48) & 0,73^{*} \\ 34(69,4) & 14(77,8) & & \\ & & & \\ 18(36,7) & 11(61,1) & 0,37(0,12-1,12) & 0,07 \\ 31(63,3) & 7(38,9) & & \end{array}$

Não

Dormem em aglomerados

Sim

Não

$31(63,3)$

$8(16,3) \quad 9(50,0)$

$0,19(0,06-0,64)$

0,005

Não

$41(83,7) \quad 9(50,0)$

\section{Doenças prévias}

Sim

$13(27,1) \quad 3(16,7)$

$1,86(0,46-7,48)$

$0,59 *$

Não

$35(72,9)$

$15(83,3)$

Vacina atualizada

Sim

$32(82,1) \quad 13(86,7)$

$0,7(0,13-3,84)$

$>0,99 *$

Não

IMC abaixo da média

$7(17,9) \quad 2(13,3)$

$7(14,3) \quad 6(33,3)$

$0,35(0,09-1,24)$

$40(81,7)$

$12(66,7)$

0,1

Não

Renda

Até um s.m.

$34(73,9) \quad 16(88,9)$

$0,35(0,07-1,77)$

$0,33^{*}$

Maior que um s.m.

$12(26,1) \quad 2(11,1)$

Escolaridade materna

EFC/ EMI/ EMC

$25(52,1) \quad 13(72,2)$

$\mathrm{EFI} / \mathrm{NA}$

$23(47,9) \quad 5(27,8)$

$0,42(0,13-1,36) \quad 0,23 *$

Legenda: n-número de casos; IMC - Índice de Massa Corpórea; s.m. - salário mínimo; EFC - Ao menos Ensino fundamental completo; EMI-Ensino Médio Incompleto; EMC-Ensino Médio Completo; EFI/NA - Ensino fundamental incompleto/não alfabetizados.

Fonte: Os autores.

Quanto ao histórico de doenças, 16,3\% $(8 / 49)$ dos indivíduos informaram que apresentavam alguma comorbidade observada desde o nascimento, como hemoglobinopatias $(n=2)$, autismo $(n=1)$, refluxo $(n=1)$, estenose pulmonar $(n=1)$, rinite alérgica $(n=1)$, transtorno da fala $(n=1)$ e nódulo na cabeça (sic) $(n=1)$. Sintomas ou doenças nos últimos 30 dias foram relatados por $26,5 \%$ (13/49) dos participantes, dos quais foram reportados: gripe $(n=3)$, febre $(n=3)$, dor abdominal $(n=3)$, dor de ouvido e febre $(n=1)$, infecção intestinal $(n=1)$, febre e vômito $(n=1)$, vômito, náusea, dor abdominal e febre $(n=1)$.

Em relação ao status vacinal e/ou falta de suplementação com vitamina $A, 14,3 \%$ (7/49) dos participantes estavam com esquema incompleto ou desatualizado para Rotavírus ( $n=3$ ); Papiloma Vírus Humano - HPV ( $n=1)$, Varicela $(n=1)$; Vacina Pneumocócica 10-Valente - PCV10 (n=1), Meningocócica sorogrupo $C(n=1)$ e Vitamina A $(n=5)$. 
A análise bivariada identificou valor de $p<0,05$ para a variável comorbidades.

Os resultados de colonização nasal, em orofaringe e ambos os sítios anatômicos de acordo com as variáveis utilizadas estão representados na tabela 2 .

Tabela 2 - Colonização por Staphylococcus aureus nasal versus colonização orofaringe e em ambos os sítios anatômicos de crianças e adolescentes sadios, Vitória da Conquista-Bahia

\begin{tabular}{|c|c|c|c|}
\hline \multirow[b]{2}{*}{ Variáveis } & \multicolumn{3}{|c|}{ Colonização por S.aureus } \\
\hline & $\begin{array}{l}\text { Nasal } \\
\mathrm{n}(\%)\end{array}$ & $\begin{array}{c}\text { Orofaringe } \\
n(\%)\end{array}$ & $\begin{array}{c}\text { Ambos os sítios } \\
n(\%)\end{array}$ \\
\hline \multicolumn{4}{|l|}{ Sexo } \\
\hline Feminino & $27(60,0)$ & $9(37,5)$ & $8(40,0)$ \\
\hline Masculino & $18(40,0)$ & $15(62,5)$ & $12(60,0)$ \\
\hline \multicolumn{4}{|l|}{ Idade (em anos) } \\
\hline$\leq 11$ & $39(86,7)$ & $22(91,7)$ & $18(90,0)$ \\
\hline$\geq 12$ & $6(13,3)$ & $2(8,3)$ & $2(10,0)$ \\
\hline \multicolumn{4}{|l|}{ Cor } \\
\hline Branco & $14(31,1)$ & $8(33,3)$ & $8(40,0)$ \\
\hline Não branco & $31(68,9)$ & $16(66,7)$ & $12(60,0)$ \\
\hline $\begin{array}{l}\text { Convívio com } \\
\text { crianças }\end{array}$ & & & \\
\hline Sim & $21(46,7)$ & $10(41,6)$ & $9(45,0)$ \\
\hline Não & $24(53,3)$ & $14(58,8)$ & $11(55,0)$ \\
\hline \multicolumn{4}{|c|}{ Dormem aglomerados } \\
\hline Sim & $15(33,3)$ & $9(37,5)$ & $6(30,0)$ \\
\hline Não & $30(66,7)$ & $15(62,5)$ & $14(70,0)$ \\
\hline \multicolumn{4}{|l|}{ Doença prévia } \\
\hline Sim & $13(28,9)$ & $7(29,1)$ & $6(30,0)$ \\
\hline Não & $32(71,1)$ & $17(70,9)$ & $14(70,0)$ \\
\hline \multicolumn{4}{|c|}{ Status vacinal atualizado } \\
\hline Sim & $30(83,3)$ & $16(66,7)$ & $14(77,8)$ \\
\hline Não & $6(16,7)$ & $5(33,3)$ & $4(22,2)$ \\
\hline \multicolumn{4}{|l|}{ Renda } \\
\hline Menor que um s.m. & $31(73,8)$ & $18(78,3)$ & $15(78,9)$ \\
\hline Maior que um s.m. & $11(26,2)$ & $5(21,7)$ & $4(21,0)$ \\
\hline \multicolumn{4}{|l|}{ Escolaridade materna } \\
\hline $\mathrm{EFI} / \mathrm{NA}$ & $25(55,6)$ & $10(41,7)$ & $9(45,0)$ \\
\hline EFC/ EMI/ EMC & $20(44,4)$ & $14(58,3)$ & $11(55,0)$ \\
\hline \multicolumn{4}{|l|}{ Uso de antibióticos } \\
\hline Sim & $36(92,3)$ & $2(10,0)$ & $16(88,9)$ \\
\hline Não & $3(7,7)$ & $19(95,0)$ & $2(11,1)$ \\
\hline
\end{tabular}

Legenda: n-número de casos; s.m. - salário mínimo; EFI/NA - Ensino fundamental incompleto/não alfabetizados; EFC - Ao menos Ensino fundamental completo; EMI-Ensino Médio Incompleto; EMCEnsino Médio Completo.

Fonte: Os autores. 
0 perfil de susceptibilidade antimicrobiana foi realizado para 66,7\% (30/45) dos isolados das narinas e para 70,8\% (17/24) das amostras orofaríngeas. A respeito da resistência a uma droga e/ou múltiplas drogas, o gráfico 1 representa os dados com relação à resistência antimicrobiana intermediária e total. Entre os isolados de colonização nasal, foi identificada resistência a pelo menos um antimicrobiano para $26,7 \%(n=8)$ dos casos, sendo a maior taxa para eritromicina $(23,3 \% ; n=7)$ seguido de $6,7 \%(n=2)$ para oxacilina e $3,3 \%(n=1)$ para cada um dos antimicrobianos clindamicina, tetraciclina, sulfametoxazol/trimetoprim e gentamicina.
Apresentaram resistência a dois ou mais antibióticos, $37,5 \%(n=3)$ dos casos. Para os isolados de orofaringe foi identificada não susceptibilidade para eritromicina em 35,3\% $(n=6)$ dos casos. Para os demais antimicrobianos testados, os isolados foram susceptíveis. Nesta avaliação, foi determinado o fenótipo de resistência MLSB (resistência aos macrolídeos, lincosaminas e estreptograminas) para $13,6 \%$ $(n=6)$ entre as amostras nasais e de $29,4 \%(n=5)$ para os isolados de orofaringe.

Gráfico 1 - Prevalência de resistência aos antimicrobianos para Staphylococcus aureus isolados de amostra nasal e de orofaringe

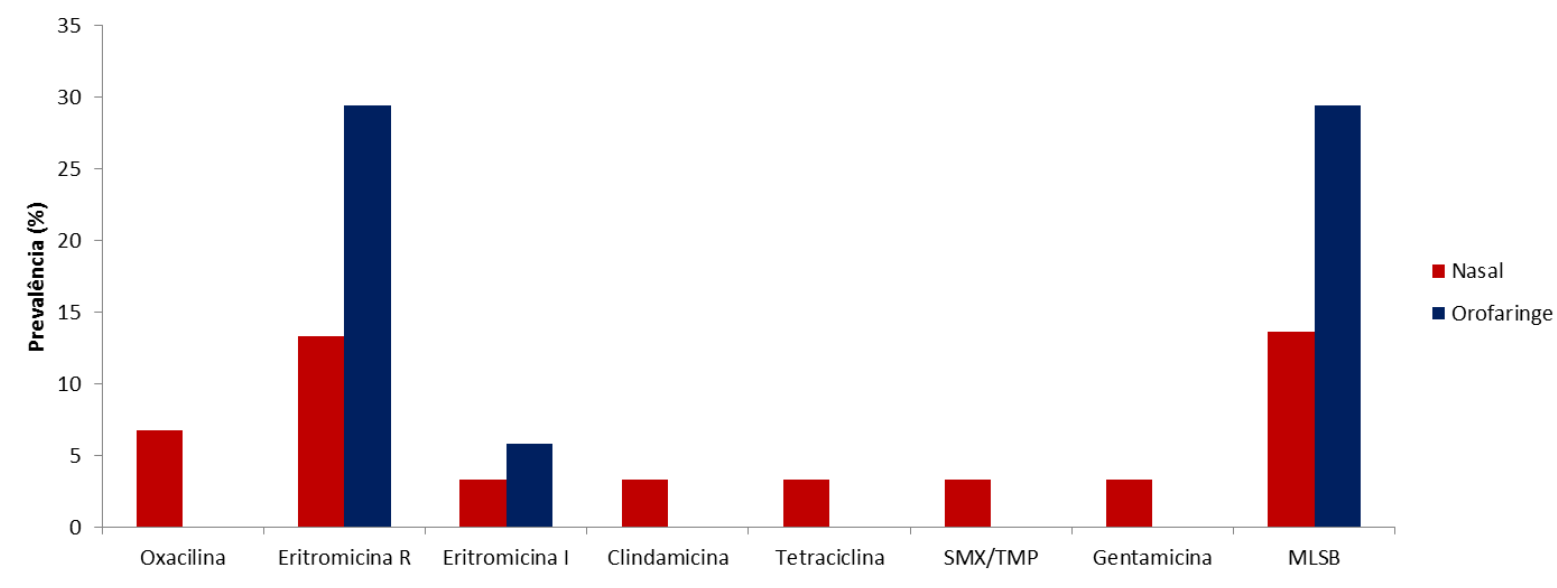

Legenda: R=Resistente; $\quad \mathrm{I}=$ Sensível aumentando a exposição; SMX/TMP=Sulfametoxazol/trimetoprim; MLSB=Fenótipo de resistência aos macrolídeos, lincosaminas e estreptograminas.

Fonte: Os autores 


\section{Discussão}

Estima-se que a prevalência média de colonização assintomática por $S$. aureus na população geral varie entre 20 e $40 \%{ }^{6,14}$. Estudo realizado com crianças na Nova Zelândia revelou que a prevalência de $S$. aureus está entre as mais altas relatadas no mundo, sendo mais frequente em populações que sofrem várias formas de privação como: renda, propriedade familiar, ocupação, níveis de emprego, educação e acesso a telecomunicações ${ }^{10}$. No Brasil, não há sistematização dos dados de colonização, contudo um estudo reportou prevalência de colonização estafilocócica de $9,1 \%$ entre neonatos e de $20,1 \%$ entre crianças atendidas em ambulatório de pediatria em uma cidade brasileira com alto consumo de antimicrobianos ${ }^{18}$ .Entretanto, nesse estudo foi encontrada taxa de $73,1 \%$, encontrado em outros trabalhos que também analisaram crianças saudáveis e obtiveram prevalências variando entre $56 \%$ e $60 \%{ }^{14,19}$. Esse cenário de discrepância pode ser devido ao pequeno tamanho amostral e também à homogeneidade dessa população.

No entanto, o presente trabalho encontrase alinhado com os resultados obtidos por Patil et al. ${ }^{1}$ cujos dados revelam que as crianças em idade escolar, do sexo masculino e autodeclarados não brancos são o principal reservatório da bactéria, porém a prevalência de colonização pode ser alterada com a idade, sendo a maior taxa encontrada entre indivíduos com quatro anos e o decréscimo a partir dos 11 anos. Esse resultado pode ser reflexo da área geográfica e localização do estudo, visto que bairros e comunidades brasileiras com vulnerabilidade social possuem maior população não branca ${ }^{20}$, e segundo censo do Instituto Brasileiro de Geografia e Estatística ${ }^{16}$, a população do bairro em que o estudo foi realizado era de 2.162 habitantes, dos quais $74,7 \%$ eram autodeclarados não brancos (pretos ou pardos) e entre as crianças e adolescentes de 5 a 14 anos essa porcentagem era de 23,4\%. Ainda que os resultados dessas variáveis não apresentem relevância estatística $(p=0,51)$, são informações importantes para traçar os aspectos epidemiológicos de S.aureus e orientar medidas de prevenção a infecções ${ }^{21}$.

No que tange aos nichos extranasais de S.aureus, estudos têm destacado a orofaringe como importante ou até mais frequente para a colonização ${ }^{6,14,19}$. Estudo avaliou crianças neozelandesas e identificou frequência de 41,1\% de colonização estafilocócica na orofaringe e de $31,5 \%$ nas narinas ${ }^{14}$. 0 presente estudo encontrou taxa menor para orofaringe em relação à nasal $(37,5 \%$ e $67,2 \%$, respectivamente). Embora este resultado esteja abaixo do esperado, pois as atividades educacionais e recreativas nesta comunidade envolvem contato direto entre os participantes, como o compartilhamento de instrumentos musicais, de materiais para alimentação, como pratos, copos e talheres, bem como de roupas para as atividades, sabe-se que o estado de portador por ser um evento dinâmico, sofre variações. Estes ocorrem conceitualmente sob dois aspectos: o temporal, segundo o qual a colonização poderia existir de forma persistente, transitória ou intermitente e o da diversidade genotípica, que considera a possibilidade de alternância das cepas colonizadoras ${ }^{22}$.

Quando não são realizadas pelo menos três coletas por paciente, perde-se a oportunidade de detecção em $20 \%$ dos indivíduos verdadeiramente colonizados ${ }^{23}$. Este aspecto pode ter influenciado na colonização nasal e/ou orofaríngea deste estudo, pois foi realizada apenas uma coleta. Em adição, não foi possível coletar amostras orofaríngeas de $4 \%$ das crianças $(n=3)$ que poderiam estar colonizadas, provocando alteração dos números, mas sem alterar o destaque da colonização nasal.

A prevalência de colonização dos dois nichos também apresentou discrepância com o estudo neozelandês ${ }^{14}$ (15,9\% versus $\left.31,2 \%\right)$, mas foi compatível com o estudo mexicano ${ }^{19}$ que apresentou $39,8 \%$. Essas diferenças podem ser justificadas pelo tamanho amostral e/ou comunidade estudada, devido às variações de exposição, condições geográficas e demográficas, aspectos socioeconômicos e sanitários e faixa etária alvo, considerando que o Brasil e o México apresentam mais aspectos semelhantes em detrimento da Nova Zelândia, ainda que existam desigualdades neste país.

Relacionado aos aspectos socioeconômicos e sanitários, estudos destacam que indivíduos que dormem ou convivem em aglomerados apresentam um fator que favorece a transmissão e colonização da bactéria por meio do compartilhamento de lençóis e outros utensílios que tenham contato com a pele. Acredita-se também que indivíduos da mesma família tendem a carrear cepas geneticamente semelhantes, sugerindo transmissão horizontal ${ }^{24}$. Em nosso estudo, uma amostragem maior, provavelmente, levaria a uma estimativa melhor do parâmetro populacional para esta variável.

Em relação às comorbidades, os dados sugerem que as crianças que apresentam alguma condição clínica sejam mais bem cuidadas desde 
o nascimento, o que poderia implicar em menor taxa de colonização ou um viés devido ao tamanho amostral. Quanto às doenças prévias dos analisados, estas não tiveram relevância estatística comprovada para estabelecimento da colonização, tendo em vista que os mais acometidos foram os indivíduos que não as apresentaram. Todavia, cabe ressaltar que pacientes imunocomprometidos e colonizados tornam-se susceptíveis ao desenvolvimento de doenças que vão desde infecções superficiais de pele a doenças invasivas com risco de morte ${ }^{6,24}$.

Poucas pesquisas avaliam a relação status vacinal e suplementação de vitamina $A$ ao processo de colonização por S. aureus. Estudo experimental conduzido com ratos comprovou que a deficiência desta vitamina provoca fragilização do sistema imune ocasionando artrite ou morte dos animais ${ }^{25}$. Outros autores (2018) ${ }^{26}$ avaliaram o sinergismo entre vitaminas e antimicrobianos diversos e embora a ação sinérgica da Vitamina A não esteja totalmente esclarecida, foi comprovado o efeito benéfico das vitaminas $\mathrm{E}$ e $\mathrm{K}$.

Dessa forma, ainda que não se possa inferir de forma isolada que a ausência de suplementação da vitamina A seja um fator de risco, pode-se sugerir que este fator aliado a variáveis como baixa renda familiar $(78,1 \%$ dos participantes do estudo tem renda de até um salário mínimo) e má qualidade da alimentação (20,9\% apresentaram IMC abaixo do esperado para a idade) podem ter influenciado na colonização. Sendo assim, a desnutrição ou nutrição deficiente decorrente de uma dieta não balanceada e diversificada, majoritariamente observada nas populações de baixa renda ${ }^{27}$, pode proporcionar uma fragilidade imunológica, bem como, ausência de efeitos sinérgicos com antimicrobianos, culminando em colonização persistente e/ou desenvolvimento de doenças.

Quanto às estratégias de imunoprevenção, embora não esteja disponível vacina estafilocócica, a exemplo das pneumocócicas implementadas no calendário vacinal do Brasil e em outros países, o microbioma pode ainda assim ser afetado. Este se torna mais complexo, fortalece o sistema imune e os efeitos antimicrobianos das bactérias comensais, além de reduzir e prevenir infecções por outras espécies bacterianas ${ }^{28}$.

Neste estudo, não foi possível estabelecer correlação sobre o impacto do tabagismo passivo nas crianças e a colonização. Em uma revisão bibliográfica, não foi obtido consenso quanto à participação do tabaco na colonização bacteriana, sendo necessários mais estudos para

avaliar se o convívio com fumantes teria alguma associação $^{24}$.

Os participantes deste estudo, em sua maioria, fazem parte das famílias com baixa renda. Embora não tenha sido identificada associação estatística significante entre colonização e esta variável $(p=0,33)$, sabe-se que o estado de portador pode sofrer influências de outros fatores biopsicossociais que circundam os indivíduos envolvidos. Uma pesquisa brasileira ${ }^{29}$ avaliou as diferenças de colonização bacteriana causada por S.aureus e Streptococcus betahemolítico em crianças avaliadas conforme categorias socioeconômicas. Foi evidenciado que crianças da classe média/baixa foram as mais colonizadas e, curiosamente, morar em favelas não foi associado como um fator de risco para essa colonização, mas sim morar em seus arredores. Os autores consideraram que estas crianças por geralmente estarem em contato com zonas industriais e comerciais, estariam mais susceptíveis à transmissão.

Autores de um estudo brasileiro (2009) ${ }^{30}$ observaram que o maior nível de escolaridade materna é um fator de proteção para a colonização, porém no estudo, os dados que informam sobre a prevalência entre os colonizados cujas mães apresentam melhor escolaridade não apresentaram significância estatística $(p=0,23)$. Ainda assim, pode-se inferir que o nível de escolaridade é um fator relacionado ao nível socioeconômico. Mesmo que haja alguma instrução materna, a conformidade com as medidas de controle que minimizam a propagação de S.aureus podem não ser executadas idealmente. A ausência de condições financeiras também pode levar a condições de privação de medidas sanitárias ideais, acesso adequado à saúde, alimentação e educação continuada, a fim de se obter a prevenção da colonização e da evolução de doenças.

A interpretação do perfil de susceptibilidade aos antibióticos evidenciou elevada taxa de resistência aos macrolídeos em amostras nasais e de orofaringe, contrariando estudos de colonização por $S$. aureus em crianças de outros países, onde reportam menor ou nenhuma taxa de resistência a esta classe de antimicrobianos, maior resistência à penicilina, seguido do sulfametoxazol-trimetoprim ${ }^{31,32}$. Outros trabalhos tem identificado diferentes taxas de resistência à eritromicina $(1,7 \%$ e de $19,5 \%)^{14,33}$ e de susceptibilidade para oxacilina e penicilina $^{14}$.

Neste estudo, a deteç̧ão do fenótipo de resistência MLSB, que induz a uma falsa 
sensibilidade à clindamicina, pode desencadear falha terapêutica quando prescrição de antibiótico que não cobre essa variante. Este fato alarma a comunidade científica, que se vê restrita a poucas opções terapêuticas disponíveis para combater organismos resistentes ${ }^{34}$.

Comumente, infecções não complicadas do trato respiratório são tratadas com azitromicina ou clindamicina ${ }^{35}$, sugerindo que a resistência à eritromicina seja resultado do uso empírico e/ou antimicrobianos de amplo espectro a nível ambulatorial ou hospitalar. Diante desse cenário de resistência antimicrobiana e cepas MRSA, é necessário o constante estado de vigilância e incentivo às práticas já implementadas pela Organização Mundial de Saúde, como prescrição e uso consciente dos antibióticos, aplicação de normas que impeçam a venda destes sem prescrição médica e cumprimento de medidas de prevenção à contaminação, como lavagem de mãos e uso de álcool em gel ${ }^{36}$. Mais estudos são necessários para estabelecimento de alternativas terapêuticas e profiláticas, como a vacinação.

\section{Considerações finais}

Neste trabalho foi identificada elevada taxa de colonização nasal e orofaríngea por $S$. aureus entre as crianças e adolescentes da comunidade estudada. Dada à importância clínica desse patógeno e tendo em vista que crianças portadoras deste micro-organismo tornam-se seus propagadores, tal colonização pode implicar no desenvolvimento de doenças que variam desde afecções leves de pele a complicações graves que podem evoluir para a morte. Deste modo, evidencia-se a necessidade de contínuo monitoramento epidemiológico e clínico deste patógeno nesta comunidade, principalmente para aqueles indivíduos que já apresentam perfil de resistência antimicrobiana a fim de minimizar os impactos clínicos. Considerando que vivenciamos uma redução nas opções terapêuticas efetivas para o seu combate, ressalta-se a importância da prescrição adequada de antimicrobianos e do uso racional de medicamentos pela população.

\section{Referências}

1. Patil AK, Namineni $S$, Cheruku SR, Penmetsa C, Penmetcha S, Mallineni SK. Prevalence of Community-Associated MethicillinResistant Staphylococcus aureus in Oral and Nasal Cavities of 4 to 13-year-old Rural School Children: A Cross-sectional Study. Contemp Clin
Dent. 2019; 10(1):99-104. doi: http://dx.doi.org/10.4103/ccd.ccd 45218.

2. Vaez H, Ghalehnoo ZR. Molecular characteristics of Staphylococcus aureus nasal carriage among health care workers at a Referral Hospital in Zabol, Iran. Pan Afr Med J. 2019; 34:196. doi: http://dx.doi.org/10.11604/pamj.2019.34.196.16 645.

3. Ricardo Caldera DM, Buelvas Doria FA, Escobar Pérez JA, Tovar Acero C. Colonización y factores de virulencia de Staphylococcus aureus resistente a meticilina en uma población infantil de Montería. latreia. 2015; 28(3):259-68. doi: http://dx.doi.org/10.17533/udea.iatreia.v28n3a0 4 .

4. Pereira MFB. Colonização por Staphylococcus aureus em pediatria [Mestrado]. São Paulo: Faculdade de Ciências Médicas da Santa Casa de São Paulo; 2011. Disponível em:

http://www.fcmsantacasasp.edu.br/wpcontent/uploads/dissertacoes-e-teses/cienciasdasaude/doutorado/2011/2011 Maria Fernanda Badue Pereira.pdf

5. Bernhard K, Christopher W, Alexander Z, Andreas $P$. The commensal lifestyle of Staphylococcus aureus and its interactions with the nasal microbiota. Nature Reviews Microbiology. 2017; 15: 675-687. doi: http://dx.doi.org/10.1038/nrmicro.2017.104.

6. Claudia L, Andreas P, Bernhard K. Staphylococcus aureus Coloniza ín of the Human Nose and Interaction with Other Microbiome Members. Microbiology Spectrum. 2019; 7(2):1-10. doi: http://dx.doi.org/10.1128/microbiolspec.GPP30029-2018.

7. Rachel J. G, Franklin D. Lowy. Pathogenesis ofmethicillin-resistant Staphylococcus aureus infection. Clin Infect Dis. 2008; 46(5):350-359. doi: https://doi.org/10.1086/533591.

8. Cassettari VC, Strabelli T, Medeiros EAS. Staphylococcus aureus bacteremia: what is the impact of oxacillin resistance on mortality. Braz J Infect Dis. 2005; 9(I):70-6. https://doi.org/10.1590/S141386702005000100012.

9. Lima, B.P., Hu, L.I., Vreeman, G.W. et al. The Oral Bacterium Fusobacterium nucleatum Binds Staphylococcus aureus and Alters Expression of the Staphylococcal Accessory Regulator sarA. Microb Ecol. 2019; 78:336-347. doi: https://doi.org/10.1007/s00248-018-1291-0. 
10. Santos $A L$, Santos DO, Freiras CC, Ferreira BLA, Afonso IF, Rodrigues CR et.al. Staphylococcus aureus: visitando uma cepa de importância hospitalar. J Bras Patol Med. Lab. 2007; 43(6):413-423. doi: https://doi.org/10.1590/S167624442007000600005.

11. Parastan $R$, Karga $M$, Solhjoo K, Kafilzadeh F. A synergistic association between adhesion-related genes and multidrug resistance patterns of Staphylococcus aureus isolates from different patients and healthy individuals. J Glob Antimicrob Resist. 2020; Mar 10. pii: S22137165(20)30054-0.

doi: https://dx.doi.org/10.1016/j.jgar.2020.02.025.

12. Murray PR, Rosenthal KS, Pfaller MA. Microbiologia Medica. In: Capítulo 14. Mecanismos de Patogenicidade Bacteriana; Capítulo 18 Staphylococcus e Outros Cocos Gram-positivos Relacionados. 7a ed. Rio de Janeiro: Elsevier; 2014. p.259-273;318-342.

13. Hosseini $M$, Shapouri Moghaddam A, Derakhshan S, Hashemipour SMA, HadadiFishani M, Pirouzi A, Khaledi A. Correlation Between Biofilm Formation and Antibiotic Resistance in MRSA and MSSA Isolated from Clinical Samples in Iran: A Systematic Review and Meta-Analysis. Microb Drug Resist. 2020; 10 de março.

doi: https://dx.doi.org/10.1089/mdr.2020.0001.

14. Williamson DA, Ritchie $S$, Keren $B$, Harrington $\quad H$, Thomas $M G$, Upton Um, Lennon D, Leversha Uma . Persistence, Discordance and Diversity of Staphylococcus aureus Nasal and Oropharyngeal Colonization in School-aged Children. Pediatr Infect Dis J. 2016; 35

(7):744-8.

doi: https://dx.doi.org/10.1097/INF.0000000000 001173.

15. Ossa R, Prado SI, Ceci MC, Lima D, Martinez R, Belissimo-Rodrigues F. Is communityassociated methicillin-resistant Staphylococcus aureus (CA-MRSA) an emerging pathogen among children in Brazil?. Braz J Infect Dis. 2018; 22(5):371-376.

doi: https://dx.doi.org/10.1016/i.bjid.2018.10.276. 16. Instituto Brasileiro de Geografia e Estatística. IBGE. População residente, por cor ou raça, segundo a situação do domicílio, o sexo e a idade. [comunicação pessoal em 2019 setembro 20]. Comunicação pessoal enviada por: gabriel.couto@ibge.gov.br.

17. Biblioteca Virtual em Saúde. BVS. Cálculo do Índice de Massa Corporal. São Paulo, $2020 . \quad$ Disponível em: https://aps.bvs.br/apps/calculadoras/?page=7.
18. Vieira MA, Minamisava R, Pessoa-Júnior $\mathrm{V}$, Lamaro-Cardoso J, Yves MT, Porfirio AMC et al. Methicillin-resistant Staphylococcus aureus nasal carriage in neonates and children attending a pediatric outpatient clinics in Brazil. Braz J Infect Dis. 2013; 18(1):44-47. doi: https://doi.org/10.1016/i.bjid.2013.04.012.

19. Hamdan-Partida A, Sainz-Espuñes T, Bustos-Martínez J. Characterization and persistence of Staphylococcus aureus strains isolated from the anterior nares and throats of healthy carriers in a Mexican community. J Clin Microbiol. 2010; 48(5):1701-1705. doi: https://dx.doi.org/10.1128/JCM.01929-09.

20. Vieira I. IBGE: negros são $17 \%$ dos mais ricos e três quartos da população mais pobre. [citado em 2016 dezembro 02]. Disponível em: https://agenciabrasil.ebc.com.br/geral/notici a/2016-12/ibge-negros-sao-17-dos-maisricos-e-tres-quartos-da-populacao-maispobre.

21. Blumental $S$, Deplano A, Jourdain S, De Mendonça R, Hallin M, Nonhoff $C$, Rottiers $S$, Vergison A, Denis O. Dynamic pattern and genotypic diversity of Staphylococcus aureus nasopharyngeal carriage in healthy preschool children. Journal of Antimicrobial Chemotherapy. 2013; 68 (7):1517-1523.doi: https://dx.doi.org/10.1093/jac/dkt080.

22. Pan ES, Diep BA, Charlebois ED, Auerswald C, Carleton HA, Sensabaugh GF. et al. Population Dynamics of Nasal Strains of Methicillin-Resistant Staphylococcus aureus and Their Relation to Community-Associated Disease Activity. J Infect Dis. 2005;192:811-818. doi: https://dx.doi.org/10.1086/432072.

23. Padoveze MC, Pedro RJ, Blum-Menezes D, Bratifich OJ, Moretti All. Staphylococcus aureus nasal colonization in HIV outpatients persistent or transient?. Am J Infect Control. 2008; $36 \quad$ (3):187-191. doi: https://dx.doi.org/10.1016/j.ajic.2007.05.012.

24. Sakr A, Brégeon F, Mège JL, Rolain JM, Blin O. Staphylococcus aureus Nasal Colonization: An Update on Mechanisms, Epidemiology, Risk Factors, and Subsequent Infections. Front Microbiol. 2018; 9:2419. doi: https://doi.org/10.3389/fmicb.2018.02419.

25. Wiedermann U, Tarkowski A, Bremell T, Hanson LA, Kahu H, Dahlgren UI. Vitamin A deficiency predisposes to Staphylococcus aureus infection. Infect Immun. 1996; 64(1):209-214. Disponível em: https://www.ncbi.nlm.nih.gov/pubmed/8557341 26. Shahzad S, Ashraf MA , Sajid M, Shahzad A, Rafique A, Mahmood MS. Evaluation of 
synergistic antimicrobial effect of vitamins (A, B1, $B 2, B 6, B 12, C, D, E$ and $K$ ) with antibiotics against resistant bacterial strains. J Glob Antimicrob Resist. 2018; 13:231-236. doi: https://dx.doi.org/10.1016/j.jgar.2018.01.00 5.

27. Andriolli BG, Ferraz D, Oliveira FCR. O padrão de consumo alimentar das famílias brasileiras: fatores determinantes e tendências. In: XXXVII Encontro Nacional de Engenharia de Produção - ENEGEP; 2017 october 10-13. Joinville, Santa Catarina, Brasil. doi: https://dx.doi.org/10.14488/ENEGEP2017 TN ST O 24240232715 .

28. Salgado VR, Fukutani KF, Fukutani E, Lima JV, Rossi EA, Barral A, Oliveira $\mathrm{Cl}$, Nascimento-Carvalho C, Van Weyenbergh J, Queiroz ATL. Effects of 10-valent pneumococcal conjugate (PCV10) vaccination on the nasopharyngeal microbiome. Vaccine. 2020; 38(6):1436-1443.

doi: https://dx.doi.org/10.1016/j.vaccine.2019.1 1.079 .

29. Neves FPG, Marlow MA, RezendePereira G, et al. Differences in gram-positive bacterial colonization and antimicrobial resistance among children in a high income inequality setting. BMC Infect Dis. 2019; 19(1):478.

doi: https://dx.doi.org/10.1186/s12879-019-4104-2.

30. Lamaro-Cardoso J, de Lencastre H, Kipnis A, et al. Molecular Epidemiology and Risk Factors for Nasal Carriage of Staphylococcus aureus and Methicillin-Resistant S. aureus in Infants Attending Day Care Centers in Brazil. J Clin Microbiol. 2009; 47(12):3991-3997. doi: https://dx.doi.org/10.1128/JCM.01322-09.

31. Singh AK, Agarwal L, Kumar A, Sengupta C, Singh RP. Prevalência de colonização nasal de Staphylococcus aureus resistente à meticilina em escolares do distrito de Barabanki, Uttar Pradesh, Índia. J Family Med Prim Care. 2018; 7(1):162-166. doi: https://dx.doi.org/10.4103/jfmpc.jfmpc 34516. 32. Reta A, Wubie M, Mekuria G. Nasal colonization and antimicrobial susceptibility pattern of Staphylococcus aureus among preschool children in Ethiopia. BMC Res Notes. 2017; 10 (1): $46 . \quad$ doi: https://dx.doi.org/10.1186/s13104-017-3079-6.

33. Furlaneto L, Sobrinho SK, Zaniboni L, Perin CT, Hernandes ES, Oliveira MT. Incidência e determinação do perfil de sensibilidade de cepas de Staphylococcus aureus isoladas da microbiota natural das fossas nasais e orofaringe de acadêmicos do curso de enfermagem. Rev Inst
Cienc Saúde. 2005; 3(1):7-10. Disponível em: https://www.unip.br/presencial/comunicacao/p ublicacoes/ics/edicoes/2005/01 jan mar/V23 N 12005 p7-10.pdf

34. Brasil. Agência Nacional de Vigilância Sanitária. Resistência antimicrobiana é ameaça global, diz OMS [citado em 2019 novembro 18]. Disponível em: http://portal.anvisa.gov.br/noticias/Lasset publisher/FXrpx9qY7FbU/content/resiste ncia-antimicrobiana-e-ameaca-global-dizoms/219201

35. Malhotra-Kumar S, Lammens C, Coenen $\underline{S}$, Van Herck K, Goossens H. Effect of azithromycin and clarithromycin therapy on pharyngeal carriage of macrolide-resistant streptococci in healthy volunteers: a randomised, double-blind, placebo-controlled study. Lancet. 2007; 369(9560):482-90. doi: https://dx.doi.org/10.1016/S01406736(07)60235-9.

36. Sampaio OS, Sancho LG, Lago. Implementação da nova regulamentação para prescrição e dispensação de antimicrobianos: possibilidades e desafios. Cad. saúde colet. [online]. 2018; 26(1):15-22. doi: https://doi.org/10.1590/1414-

462×201800010185.

\section{Endereço para Correspondência}

Milena Soares dos Santos

Av. Edmundo Silveira Flores, n.115, Residencial Villa Espanha, EdfMadri, apt. 1003. Bairro Universidade. Vitória da Conquista-Bahia.

CEP: 45.031-155

E-mail: milenasoares.ims@gmail.com

Recebido em 22/04/2021

Aprovado em 06/12/2021

Publicado em 30/12/2021 